\title{
Complete genome sequence of Acidaminococcus fermentans type strain $\left(\mathrm{VR}^{\mathrm{T}}\right)$
}

\author{
Yun-Juan Chang ${ }^{1,2}$, Rüdiger Pukall ${ }^{3}$, Elizabeth Saunders ${ }^{4}$, Alla Lapidus ${ }^{1}$, Alex Copeland ${ }^{1}$, \\ Matt Nolan', Tijana Glavina Del Rio ${ }^{1}$, Susan Lucas ${ }^{1}$, Feng Chen ${ }^{1}$, Hope Tice ${ }^{1}$, Jan-Fang \\ Cheng $^{1}$, Cliff Han ${ }^{1,4}$, John C. Detter ${ }^{1,4}$, David Bruce ${ }^{1,4}$, Lynne Goodwin ${ }^{1,4}$, Sam Pitluck ${ }^{1}$, \\ Natalia Mikhailova ${ }^{1}$, Konstantinos Liolios ${ }^{1}$, Amrita Pati ${ }^{1}$, Natalia Ivanova ${ }^{1}$, Konstantinos \\ Mavromatis ${ }^{1}$, Amy Chen ${ }^{5}$, Krishna Palaniappan ${ }^{5}$, Miriam Land ${ }^{1,2}$, Loren Hauser ${ }^{1,2}$, Cynthia D. \\ Jeffries $^{1,2}$, Thomas Brettin ${ }^{1,2}$, Manfred Rohde ${ }^{6}$, Markus Göker ${ }^{3}$, James Bristow ${ }^{1}$, Jonathan A. \\ Eisen $^{1,7}$, Victor Markowitz ${ }^{5}$, Philip Hugenholtz ${ }^{1}$, Nikos C. Kyrpides ${ }^{1}$, and Hans-Peter Klenk ${ }^{3 *}$ \\ ${ }^{1}$ DOE Joint Genome Institute, Walnut Creek, California, USA \\ ${ }^{2}$ Oak Ridge National Laboratory, Oak Ridge, Tennessee, USA \\ ${ }^{3}$ DSMZ - German Collection of Microorganisms and Cell Cultures GmbH, Braunschweig, \\ Germany \\ ${ }^{4}$ Los Alamos National Laboratory, Bioscience Division, Los Alamos, New Mexico, USA \\ ${ }^{5}$ Biological Data Management and Technology Center, Lawrence Berkeley National \\ Laboratory, Berkeley, California, USA \\ ${ }^{6} \mathrm{HZI}$ - Helmholtz Centre for Infection Research, Braunschweig, Germany \\ ${ }^{7}$ University of California Davis Genome Center, Davis, California, USA \\ *Corresponding author: Hans-Peter Klenk
}

Keywords: anaerobic, mesophile, diplococcus, gastrointestinal tract, trans-aconitate degradation, glutamate fermentation, Acidaminococcaceae, Selenomonadales, Negativicutes, GEBA

Acidaminococcus fermentans (Rogosa 1969) is the type species of the genus Acidaminococcus, and is of phylogenetic interest because of its isolated placement in a genomically little characterized region of the Firmicutes. A. fermentans is known for its habitation of the gastrointestinal tract and its ability to oxidize trans-aconitate. Its anaerobic fermentation of glutamate has been intensively studied and will now be complemented by the genomic basis. The strain described in this report is a nonsporulating, nonmotile, Gram-negative coccus, originally isolated from a pig alimentary tract. Here we describe the features of this organism, together with the complete genome sequence, and annotation. This is the first complete genome sequence of a member of the family Acidaminococcaceae, and the 2,329,769 bp long genome with its 2,101 protein-coding and 81 RNA genes is part of the Genomic Encyclopedia of Bacteria and Archaea project.

\section{Introduction}

Strain VR4T $(=$ DSM 20731 = ATCC 25085 = CCUG 9996) is the type strain of the species Acidaminococcus fermentans, and the type species of the genus Acidaminococcus [1,2]. A. fermentans was originally isolated by Fuller from a pig alimentary tract [3] and included in the nomina incertae sedis. It was subsequently characterized and classified in 1969 as type strain of the then-novel genus Acidaminococcus [1]. An emendation of the description of $A$. fermentans was provided by Cook et al. in 1994 [4]. Originally, the principal physiological and taxonomically distinctive feature of the strain was its ability to use amino acids as sole source of energy for growth anaerobically [1]. Three accompanying strains (VR7, VR11, VR14) from the alimentary tract of the same pig were reported and deposited in the ATCC (American Type Culture Collection) [3]. Closely related strains belonging to the species have been isolated from humans (EF060089-91, >99.9\% sequence identity) [5] and from cow rumen [4]. Several uncultured clones were isolated from human fecal samples 
(DQ904734, DQ904735 and DQ904837, 99.8\%) [6], and from rabbit cecum (EF445291, 99.8\%) [7]. The type strain of the only other species in the genus, $A$. intestini [5] shows $95.8 \% 16 \mathrm{~S}$ rRNA sequence identity with strain VR4T, whereas the type species of the other genera of the Acidaminococcaceae are less than $91.7 \%$ identical [8]. Of the many publicly available human gut metagenomes only one (BAAV01001815, 96.1\%) [9] contained a highly similar $16 \mathrm{~S}$ rRNA gene sequence, whereas none of the environmental genomic surveys indicated any moderately related phylotypes, shedding doubt on a wide-spread occurrence of members of the species $A$. fermentans (as of February 2010). Here we present a summary classification and a set of features for A. fermentans VR4', together with the description of the complete genomic sequencing and annotation.

\section{Classification and features}

Figure 1 shows the phylogenetic neighborhood of A. fermentans VR4 ${ }^{\mathrm{T}}$ in a $16 \mathrm{~S}$ rRNA based tree. The sequences of the six copies of the16S rRNA gene in the genome of strain VR4 ${ }^{\mathrm{T}}$ differ from each other by up to five nucleotides, and differ by up to seven nucleotides from the previously published $16 \mathrm{~S}$ rRNA sequences from DSM 20731 (X78017, X77951), which contain two ambiguous base calls.

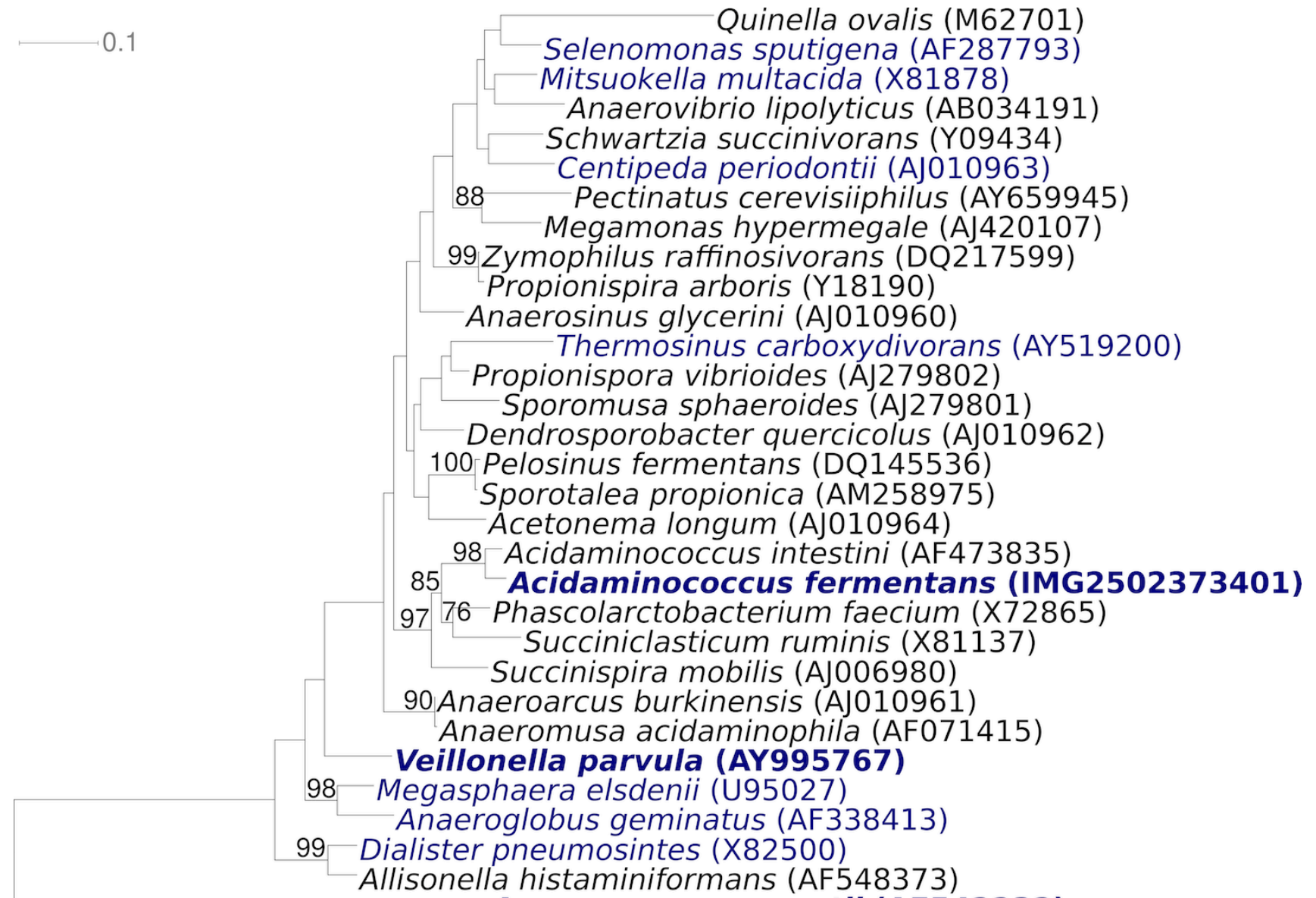

Anaerococcus prevotii (AF542232)

Figure 1. Phylogenetic tree highlighting the position of $A$. fermentans $V R 4^{\top}$ relative to the other type strain within the genus Acidaminococcus and the type strains of the other genera within the family Acidaminococcaceae. The tree was inferred from 1,348 aligned characters $[10,11]$ of the $16 \mathrm{~S}$ rRNA gene sequence under the maximum likelihood criterion [12] and rooted with the type strain of Anaerococcus prevotii, a member of the neighboring family Peptococcaceae. The branches are scaled in terms of the expected number of substitutions per site. Numbers above branches are support values from 1,000 bootstrap replicates [13] if larger than 60\%. Lineages with type strain genome sequencing projects registered in GOLD [14] are shown in blue, published genomes in bold and include. the recently published GEBA genome from A. prevotii [15] and Veillonella parvula [16]. 
Cells of $A$. fermentans strain VR4T often occur as oval or kidney-shaped diplococci that are 0.6 to $1.0 \mu \mathrm{m}$ in diameter [1] (Table 1 and Figure 2). The organism is anaerobic, nonsporulating, nonmotile, chemoorganotrophic, and Gram-negative [1]. Its optimum growth temperature is 30 to $37^{\circ} \mathrm{C}$, over a $\mathrm{pH}$ range from 6.2 to 7.5 , with an optimum at 7.0 [1]. Weak or no growth occurs at 25 and $45^{\circ} \mathrm{C}$.

Table 1. Classification and general features of $A$. fermentans VR ${ }^{\top}$ according to the MIGS recommendations [17]

\begin{tabular}{|c|c|c|c|}
\hline MIGS ID & Property & Term & Evidence code \\
\hline & \multirow{8}{*}{ Current classification } & Domain Bacteria & TAS [18] \\
\hline & & Phylum Firmicutes & TAS $[19,20]$ \\
\hline & & Class Negativicutes & TAS [21] \\
\hline & & Order Selenomonadales & TAS [21] \\
\hline & & Family Acidaminococcaceae & TAS [21] \\
\hline & & Genus Acidaminococcus & TAS $[1,2,4,5]$ \\
\hline & & Species Acidaminococcus fermentans & TAS [1] \\
\hline & & Type strain VR4 & TAS $[1,2,4]$ \\
\hline & Gram stain & negative & TAS [1] \\
\hline & Cell shape & oval; kidney shaped diplococci & TAS [1] \\
\hline & Motility & nonmotile & TAS [1] \\
\hline & Sporulation & non-sporulating & TAS [1] \\
\hline & Temperature range & mesophilic & TAS [1] \\
\hline & Optimum temperature & $30-37^{\circ} \mathrm{C}$ & TAS [1] \\
\hline & Salinity & moderate & TAS [1] \\
\hline \multirow[t]{3}{*}{ MIGS-22 } & Oxygen requirement & anaerobic & TAS [1] \\
\hline & Carbon source & glutamate & TAS [1] \\
\hline & Energy source & glutamate, citrate, trans-aconitate & TAS $[1,4]$ \\
\hline \multirow[t]{2}{*}{ MIGS-6 } & Habitat & gastrointestinal tract of homothermic animals & TAS [22] \\
\hline & $\mathrm{pH}$ & 6.2-7.5, optimum 7.0 & TAS [23] \\
\hline MIGS-15 & Biotic relationship & free-living & TAS [1] \\
\hline \multirow[t]{3}{*}{ MIGS-14 } & Pathogenicity & pathogenic for humans & TAS [24] \\
\hline & Biosafety level & 2 & TAS [24] \\
\hline & Isolation & Sus scrofa, alimentary tract & TAS $[1,3]$ \\
\hline MIGS-4 & Geographic location & not reported & NAS \\
\hline MIGS-5 & Sample collection time & about 1966 & TAS [3] \\
\hline $\begin{array}{l}\text { MIGS4. } 1 \\
\text { MIGS-4.2 }\end{array}$ & $\begin{array}{l}\text { Latitude } \\
\text { Longitude }\end{array}$ & not reported & NAS \\
\hline MIGS-4.3 & Depth & not reported & NAS \\
\hline MIGS-4.4 & Altitude & not reported & NAS \\
\hline
\end{tabular}

Evidence codes - IDA: Inferred from Direct Assay (first time in publication); TAS: Traceable Author Statement (i.e., a direct report exists in the literature); NAS: Non-traceable Author Statement (i.e., not directly observed for the living, isolated sample, but based on a generally accepted property for the species, or anecdotal evidence). These evidence codes are from the Gene Ontology project [25]. If the evidence code is IDA, then the property was directly observed for a live isolate by one of the authors or an expert mentioned in the acknowledgements. 


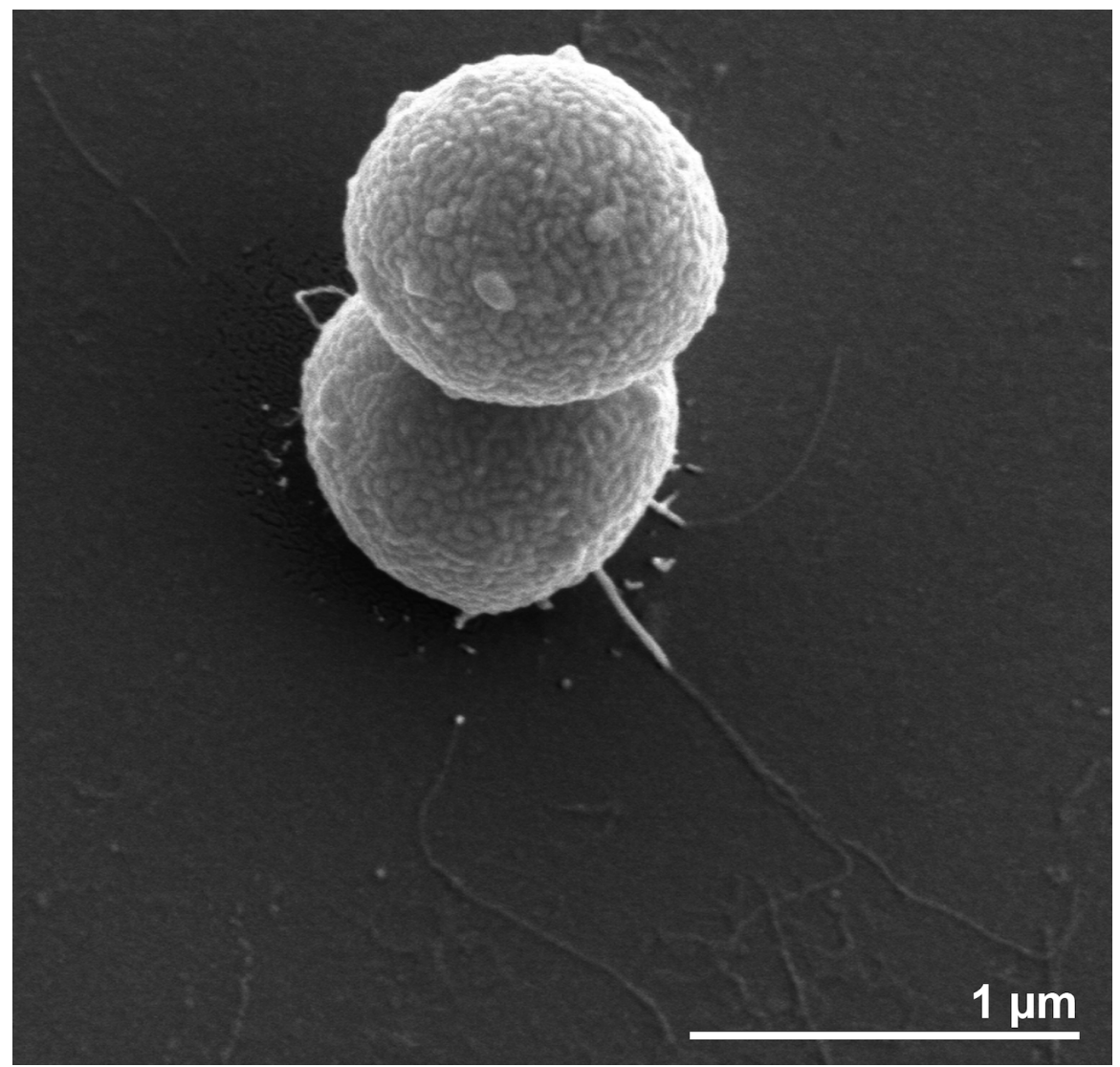

Figure 2. Scanning electron micrograph of $A$. fermentans strain VR4 ${ }^{\top}$

A. fermentans thrives mainly by glutamate fermentation via the 2-hydroxyglutarate pathway in the intestinal tract of homeothermic animals $[1,25]$, utilizing glutamate, citrate, and trans-aconitate as sole energy sources, in the presence of sodium $[4,26,27]$. Ammonia, acetate, butyrate, and hydrogen are the main end products of growth $[1,4]$. Transport and catabolism of these substrates are dependent on a sodium motive force as a method of membrane energetics $[27,28]$. The fermentation of trans-aconitate in strain VR4 ${ }^{\mathrm{T}}$ takes a different pathway, which is via citrate, oxaloacetate, and pyruvate, producing $\mathrm{CO}_{2}$, acetate, butyrate and hydrogen [26]. Unlike the citrate uptake, the aconitate uptake may adopt two mechanisms: a citrate/aconitate carrier with low affinity for sodium and an aconitate carrier with high affinity for sodium [29].

\section{Chemotaxonomy}

A. fermentans VR4 ${ }^{\mathrm{T}}$ contains meso-diaminopimelic acid in the cell wall; extracts from whole cells contain galactose, glucose and ribose. Menaquinones and ubiquinones are absent; phospholipids present are disphosphatidyl-glycerol, phosphatidylethanolamine and an additional phospholipid, possibly phosphatidylcholine [30]. Major fatty acids ( $>5 \%$ of total) are $\mathrm{C}_{16: 1 \text { cis-7 }}(30 \%), \mathrm{C}_{14: 0-30 \mathrm{H}}(15.8 \%), \mathrm{C}_{18: 1}$ cis-11 (10.2\%), $\mathrm{C}_{12: 0}(9.8 \%), \mathrm{C}_{16: 1 \text { cis-7 }}(9.5 \%)$, and $\mathrm{C}_{18: 1 \text { cis-9 }}$ (5.8\%) [30]. Monoenoic unsaturated phospholipid fatty acids (PLFAs) are dominant, indicating a Gram-negative bacterium, branched monoenoic or mid-branched saturated PLFAs as biomarker for anaerobic respiration is also prominent [31].

\section{Genome sequencing and annotation Genome project history}

This organism was selected for sequencing on the basis of its phylogenetic position [32], and is part of the Genomic Encyclopedia of Bacteria and Archaea project [33]. The genome project is deposited in the Genomes OnLine Database [14] and the complete genome sequence in GenBank. Sequencing, finishing and annotation were performed by the DOE Joint Genome Institute (JGI). A summary of the project information is shown in Table 2. 
Table 2: Genome sequencing project information

\begin{tabular}{lll}
\hline MIGS ID & \multicolumn{1}{c}{ Property } & \multicolumn{1}{c}{ Term } \\
\hline MIGS-31 & Finishing quality & Finished \\
MIGS-28 & Libraries used & One genomic 8kb pMCL200 library, one 454 pyrosequence \\
library and one Illumina library & ABI3730, 454 Titanium, Illumina GA \\
MIGS-29 & Sequencing platforms & $5 \times$ Sanger; 58.3× pyrosequence \\
MIGS-31.2 & Sequencing coverage & Newbler version 2.0.0-PostRelease-11/04/2008, phrap \\
MIGS-30 & Assemblers & Prodigal, GenePRIMP \\
MIGS-32 & Gene calling method & CP001859 \\
& INSDC ID & January 19, 2010 \\
& Genbank Date of Release & Gc01187 \\
& GOLD ID & 33685 \\
& NCBI project ID & 2502171195 \\
& Database: IMG-GEBA & DSM 20731 \\
MIGS-13 & Source Material Identifier & Tree of Life, GEBA \\
& Project relevance &
\end{tabular}

\section{Growth conditions and DNA isolation}

A. fermentans VR4T, DSM 20731, was grown under strictly anaerobic conditions in DSMZ medium 414 [34] at $37^{\circ} \mathrm{C}$. DNA was isolated from 1-1.5 g of cell paste using Qiagen Genomic 500 DNA Kit (Qiagen, Hilden, Germany) following the manufacturer's instructions with modification st/LALMP for cell lysis according to Wu et al. [33].

\section{Genome sequencing and assembly}

The genome was sequenced using a combination of Sanger and 454 sequencing platforms. All general aspects of library construction and sequencing can be found at http://www.jgi.doe.gov/. 454 Pyrosequencing reads were assembled using the Newbler assembler version 2.0.0-PostRelease11/04/2008 (Roche). Large Newbler contigs were broken into 2,561 overlapping fragments of 1,000 bp and entered into the final assembly as pseudoreads. The sequences were assigned quality scores based on Newbler consensus q-scores with modifications to account for overlap redundancy and to adjust inflated q-scores. A hybrid 454/Sanger assembly was made using the parallel phrap assembler (High Performance Software, LLC). Possible mis-assemblies were corrected with Dupfinisher or transposon bombing of bridging clones [35]. Gaps between contigs were closed by editing in Consed, custom primer walk or PCR amplification. A total of 256 Sanger finishing reads were produced to close gaps, to resolve repetitive regions, and to raise the quality of the finished sequence. Illumina reads were used to improve the final consensus quality using an in-house developed tool (the Polisher). The error rate of the completed genome sequence is less than 1 in 100,000 . Together all sequence types provided $63.3 \times$ coverage of the genome. The final assembly contains 22,991 Sanger and 557,705 pyrosequencing reads.

\section{Genome annotation}

Genes were identified using Prodigal [36] as part of the Oak Ridge National Laboratory genome annotation pipeline, followed by a round of manual curation using the JGI GenePRIMP pipeline [37]. The predicted CDSs were translated and used to search the National Center for Biotechnology Information (NCBI) nonredundant database, UniProt, TIGRFam, Pfam, PRIAM, KEGG, COG, and InterPro databases. Additional gene prediction analysis and manual functional annotation was performed within the Integrated Microbial Genomes Expert Review (IMG-ER) platform [38].

\section{Genome properties}

The genome is 2,329,769 bp long and comprises one circular chromosome with a $55.8 \%$ GC content (Table 3 and Figure 3). Of the 2,182 genes predicted, 2,101 were protein-coding and 81 were RNAs; 75 pseudogenes were also identified. The majority of protein-coding genes $(75.3 \%)$ were assigned a putative function while the remaining ones were annotated as hypothetical proteins. The 
properties and the statistics of the genome are summarized in Table 3. The distribution of genes into COGs functional categories is presented in Table 4.

\section{Insights from the genome sequence}

Different from most organisms for which the type strain genomes have so far been described in the GEBA series, $A$. fermentans strain VR4T is biochemically well described. The strain has been intensively studied for many years. Here, we describe the genomic location of the genes for the biochemically characterized enzymes, as well as the annotation of the genome using bioinformatic approaches, which may reveal additional physiological properties of the organism.

\section{Glutmate fermentation via 2-hydroxyglutarate}

The ability of $A$. fermentans to use amino acids as the sole energy source for growth is a well known characteristic, with glutamic acid being the most important amino acid for the organism [1,4]. Strain VR4T ferments glutamate via the 2-hydroxyglutarate pathway, in which, glutamate is converted to a key intermediate - (R)-2-hydroxyglutaryl-CoA, which is dehydrated to glutaconyl-CoA, followed by decarboxylation to crotonyl-CoA, then to ammonia, $\mathrm{CO}_{2}$, acetate, butyrate and hydrogen. An unusual dehydratase contains an $[4 \mathrm{Fe}-4 \mathrm{~S}]^{2+}$ cluster - acting as an activator or initiator of dehydration, is activated by an ATPdependent one-electron reduction $[29,39,40]$. The extra energy produced is conserved via $\Delta \mu \mathrm{Na}^{+}$generated by the decarboxylation of glutaconyl-CoA [41].

The dehydratase system of strain VR4 ${ }^{\mathrm{T}}$ consists of two oxygen-sensitive protein components: component $\mathrm{A}$ - the activator $(\mathrm{HgdC})$ and component $\mathrm{D}$ - the actual dehydratase $(H g d A B)[29,40]$. Component $A$ has been crystallized and its structure has been determined by X-ray crystallography at 3 Å resolution [42].

The glutaconyl-CoA decarboxylase of $A$. fermentans is a biotin-dependent sodium pump, consisting of three major polypeptide subunits: biotin carrier (alpha, gcdA), carboxytransferase (beta, $\operatorname{gcdB}$ ) and carboxylase, the actual sodium pump (gamma, gcdC) [43]. There is additional small subunit (delta, gcdC), whose function is unclear [43]. Glutaconate CoA-transferase consists of two different polypeptide chains and is necessary for the decarboxylation of glutaconate [44].

The hydroxyglutarate operon has been experimentally studied [45] and all encoding genes are annotated in the genome (Figure 4).

Table 3: Genome Statistics

\begin{tabular}{lrr}
\hline Attribute & Value & \% of Total \\
\hline Genome size (bp) & $2,329,769$ & $100.00 \%$ \\
DNA coding region (bp) & $2,096,198$ & $89.97 \%$ \\
DNA G+C content (bp) & $1,301,006$ & $55.84 \%$ \\
Number of replicons & 1 & \\
Extrachromosomal elements & 0 & \\
Total genes & 2,182 & $100.00 \%$ \\
RNA genes & 81 & $3.71 \%$ \\
rRNA operons & 6 & \\
Protein-coding genes & 2,101 & $96.29 \%$ \\
Pseudo genes & 75 & $3.44 \%$ \\
Genes with function prediction & 1,642 & $75.25 \%$ \\
Genes in paralog clusters & 283 & $13.11 \%$ \\
Genes assigned to COGs & 1,661 & $76.12 \%$ \\
Genes assigned Pfam domains & 1,724 & $79.01 \%$ \\
Genes with signal peptides & 361 & $16.54 \%$ \\
Genes with transmembrane helices & 519 & $23.79 \%$ \\
CRISPR repeats & 2 & \\
\hline
\end{tabular}




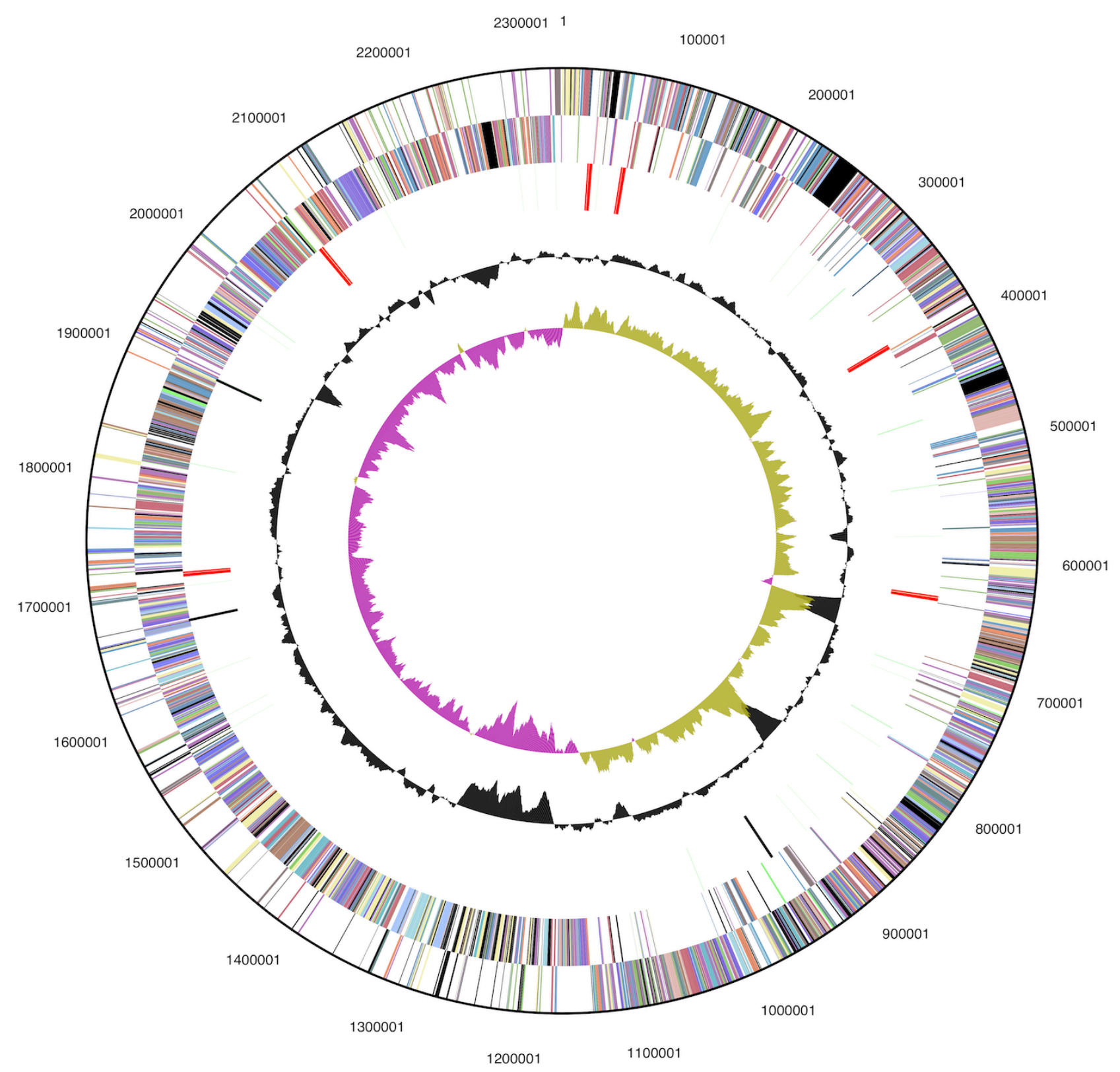

Figure 3. Graphical circular map of the genome. From outside to the center: Genes on forward strand (color by COG categories), Genes on reverse strand (color by COG categories), RNA genes (tRNAs green, rRNAs red, other RNAs black), GC content, GC skew.

The entire $7.9 \mathrm{~kb}$ long gene cluster, consists of 2hydroxyglutaryl-CoA dehydratase D-components hgdAB (Acfer_1814 and 1815), CoA-substratespecific enzyme activase (Acfer_1816), glutaconylCoA decarboxylase $\alpha$ subunit gcdA (Acfer_1817), glutaconate CoA-transferase $\alpha-$ and $\beta$-subunits (gctAB, Acfer_1819 and 1818). The glutaconyl-CoA decarboxylase $\beta-, \gamma-$ and $\delta$-subunits: $\operatorname{gcd} B, \operatorname{gcd} C$ and gcdD (Acfer_1834, Acfer_1835 and Acfer_1836) are encoded nearly $15 \mathrm{~kb}$ upstream from this operon, forming a second operon [43].
In addition to the above-mentioned protein complexes, the gene encoding glutamate dehydrogenase (NAD(P) (+)) (Acfer_1756) is encoded at the beginning of the gene cluster. Three acyl-CoA dehydrogenase genes (Acfer_1477, Acfer_1575 and Acfer_1583) were annotated at various locations, completing the pathway. Nevertheless, genes encoding 2-hydroxyglutarate dehydrogenase and Butyl-CoA:acetate CoA transferase have not yet been identified. Possibly these enzymes have additional functions in other pathways and have been annotated distinctly. 
Table 4: Number of genes associated with the general COG functional categories

\begin{tabular}{crrl}
\hline Code & Value & \%age Description \\
\hline J & 139 & 6.6 Translation, ribosomal structure and biogenesis \\
A & 0 & 0.0 RNA processing and modification \\
K & 128 & 6.1 Transcription \\
L & 119 & 5.7 Replication, recombination and repair \\
B & 1 & 0.0 Chromatin structure and dynamics \\
D & 21 & 1.0 Cell cycle control, mitosis and meiosis \\
Y & 0 & 0.0 Nuclear structure \\
V & 37 & 1.8 Defense mechanisms \\
T & 62 & 3.0 Signal transduction mechanisms \\
M & 110 & 5.2 Cell wall/membrane biogenesis \\
N & 7 & 0.3 Cell motility \\
Z & 0 & 0.0 Cytoskeleton \\
W & 0 & 0.0 Extracellular structures \\
U & 38 & 1.8 Intracellular trafficking and secretion \\
O & 55 & 2.6 Posttranslational modification, protein turnover, chaperones \\
C & 114 & 5.4 Energy production and conversion \\
G & 72 & 3.4 Carbohydrate transport and metabolism \\
E & 204 & 9.7 Amino acid transport and metabolism \\
F & 58 & 2.8 Nucleotide transport and metabolism \\
H & 79 & 3.8 Coenzyme transport and metabolism \\
I & 47 & 2.2 Lipid transport and metabolism \\
P & 92 & 4.4 Inorganic ion transport and metabolism \\
Q & 16 & 0.8 Secondary metabolites biosynthesis, transport and catabolism \\
R & 240 & 11.4 General function prediction only \\
S & 145 & 6.9 Function unknown \\
- & 521 & 24.8 Not in COGs \\
\hline & &
\end{tabular}

\section{Overview on transport systems}

About 310 putative transporter genes are annotated in the genome of strain VR4T ${ }^{\mathrm{T}}$, which constitute roughly $15 \%$ of the coding genes. The majority of these transporters belong to two groups: secondary carriers and the ATP-binding cassette ABC-type carriers. The most frequent class of ABC-type transport proteins are for importing iron/metal ions and amino acids, as shown in Table 5 .

Among all the ABC-type transport systems, 41\% are related to the transport of iron or other metal ions, comprising the largest $\mathrm{ABC}$ transporter group annotated. Additionally, two ferrous iron uptake (FeoB) systems (5 genes; TC\#9.A.8) were annotated, which are involved in $G$ protein coupled $\mathrm{Fe}^{2+}$ transport. At least 15 other gene products are involved in iron or magnesium transport and heavy metal transport and detoxification. Presumably, Acidaminococcus has adapted the ability to sequester iron from the host as a survival strategy. The abundance of this particular group of transporter genes might suggest diverse mechanisms evolved in order to compete for the limited iron supply in the gastrointestinal environment.

Figure 5 shows a comparison of the hydroxyglutarate operon among various organisms. The positional gene cluster is conserved in the two strains belonging to the genus Acidaminococcus (A. fermentans strain VR4 ${ }^{\mathrm{T}}$ and $A$. intestini strain D21), as well as in the two clostridia, whereas Fusobacterium differ slightly. 
The second most abundant $\mathrm{ABC}$ transporter group consists of amino acid transporters, followed by multi drug or antimicrobial efflux pumps (Table $5)$. This trend is visible in secondary carrier proteins; more than 18 and 26 genes encoded for amino acid transport and multi drug or antimicrobial efflux pumps, respectively. At least six genes are annotated as encoding a $\mathrm{Na}^{+}$:glutamate symporter (TC\# 2.A.27.1). This corroborates the most prominent physiological characteristic of the organism, namely that glutamate is the most important energy and carbon source. No functional sugar transport protein was identified, indicating that this organism does not utilize sugar.

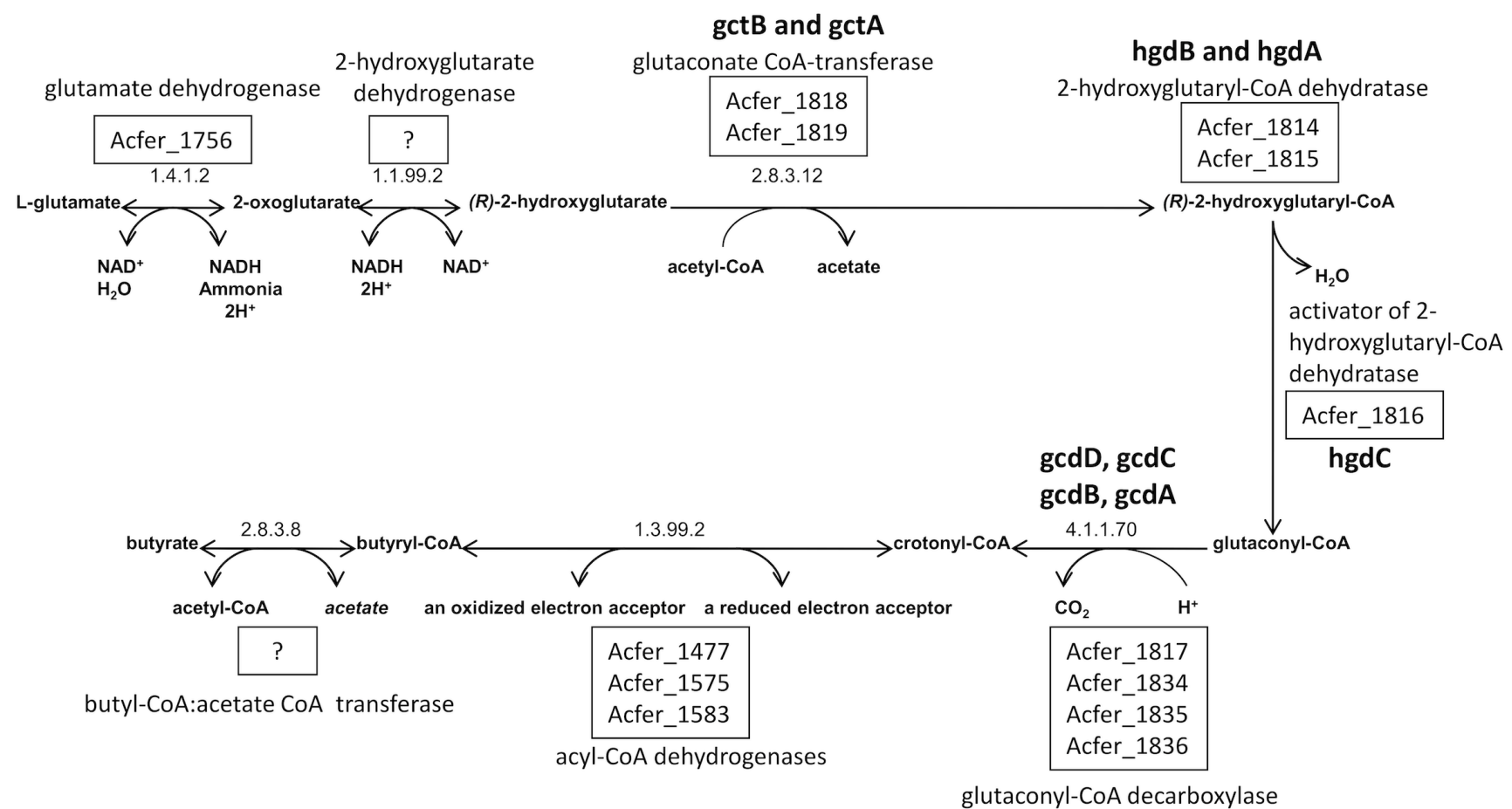

Figure 4. MetCyc pathway [46] along with the gene locus names, representing the enzymes identified in the pathway.

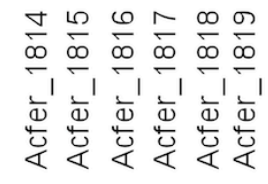

Acidaminococcus fermentans VR4 Contig169

Acidaminococcus sp. D21 Contig1_36

Clostridum sp. M62 Contig11.6

Clostridum sp. saccharolyticum-like K10

Fusobacterium nucleatum subsp. nucleatum

Fusobacterium sp. D11
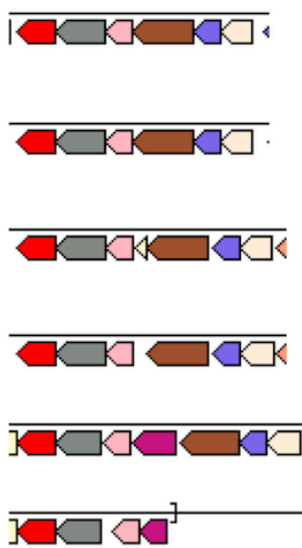

2-hydroxyglutaryt-CoA dehydratase

2-hydroxyglutary/CoA

dehydratase D-component

Activator of 2-hydroxyglutary-

CoA dehydratase

glutaconyl-CoA decarboxylase alpha subunit

glutaconate CoA-transferase subunit B

glutaconate CoA-transferase subunit A

Figure 5. Gene ortholog neighborhoods of hydroxyglutarate operons Colors indicate ortholog groups. The size of the bar approximately corresponds to gene size. Date are taken from [47] 
Table 5. Overview of the ABC-type transporters within the genome of $A$. fermentans VR4 ${ }^{\top}$

\begin{tabular}{lcc}
\hline ABC-type transporter & complete system/genes & incomplete system/genes \\
\hline ABC-type organic nutrient uptake systems & & \\
--ABC amino acid transporter & $3 / 13$ & $1 / 1$ \\
--ABC spermidine/putrescine transporter & $1 / 4$ & $0 / 0$ \\
& & \\
ABC-type inorganic nutrient uptake systems & $9 / 29$ & $3 / 3$ \\
--ABC iron uptake and other metal ion transporter & $1 / 3$ & $0 / 0$ \\
-- ABC nitrate/sulfonate/bicarbonate transporter & $2 / 5$ & $0 / 0$ \\
-- ABC phosphate transporter & & \\
& & \\
ABC-type efflux pumps & $4 / 9$ & $1 / 1$ \\
-- ABC multidrug transporter & $0 / 0$ & $0 / 0$ \\
-- ABC lipid A exporter & $1 / 2$ & $3 / 3$ \\
-- ABC antimicrobial peptide transporter & & \\
& $2 / 4$ & \\
uncharacterized ABC-type systems & & \\
\hline
\end{tabular}

The numbers represent the number of the $A B C$ system or the number of the genes involved, respectively. A functional $\mathrm{ABC}$ system, containing all necessary subunits, is referred to as complete system, otherwise it is labeled as incomplete.

Transporters for carboxylate are also noticeable. For example, the tripartite ATP-independent periplasmic transporter (TRAP-T) family (TC\# 2.A.56) is involved in the uptake of widely divergent compounds, mostly carboxylate derivatives [48]. Five TRAP systems are found in the genome, including 12 genes, one TAXI type system and four DctPQM systems. The abundance of the TRAP-T proteins is indicative of the capability of this organism to import carboxylate derivatives such as those produced by the host metabolism or fermentation by rumen microbiota, thus constituting a recycled food web and a beneficial nutritional cycle.

The Bile Acid: $\mathrm{Na}^{+}$symporter (TC\# 2.A.28) previously identified in intestinal, liver and kidney tissues of animals is identified at various locations within the genome (Acfer_0208, Acfer_0775 and Acfer_1270). This might be indicate horizontal gene transfer (HGT) between the host and $A$. fermentans VR4T. It has been shown that the acquisition of eukaryotic genes in bacteria is frequently the result of a transfer from the host [49]. Given the environmental niche of $A$. fermentans, hostmediated HGT might well have occurred.

No genes for flagellar machinery (TC\#3.A.6) are encoded in the genome, which is consistent with the observation of non-motility. A. fermentans
VR4T probably uses a type II secretion (Sec system) for protein secretion, as all components of the Sec protein export system are present (SecA, SecYEG, SecDF), except for SecB, which may be functionally replaced by a different chaperone.

\section{Comparison with the genome of Acidaminococcus intestini D21}

The genome sequence of another member of the genus Acidaminococcus (A. intestini) which was isolated from an human gastrointestinal tract D21, has been partially deciphered by the Broad Institute. The unfinished yet annotated genome sequence is deposited at NCBI (ACGB00000000) and IMG-GEBA (object-ID 643886056). The 16S rRNA sequence from $A$. sp. D21 differs from the one obtained from $A$. intestini type strain ADV 255.99 (AF473835) by just three nt, but it shares only 95.86 to $96.05 \%$ sequence similarity with $A$. fermentans VR4T indicating a considerable evolutionary distance between the two species. Despite these discrepancies, the annotated genomes indicated quite a few common physiological traits.

For instance, the 2-hydroxyglutarate operon was well conserved between the two genomes, including position, structure and individual genes (Fig.5). This suggests that both species have adopted the same glutamate fermentation pathway. 
Table 6. A list of genes discussed, reflecting the physiological insights into the organism

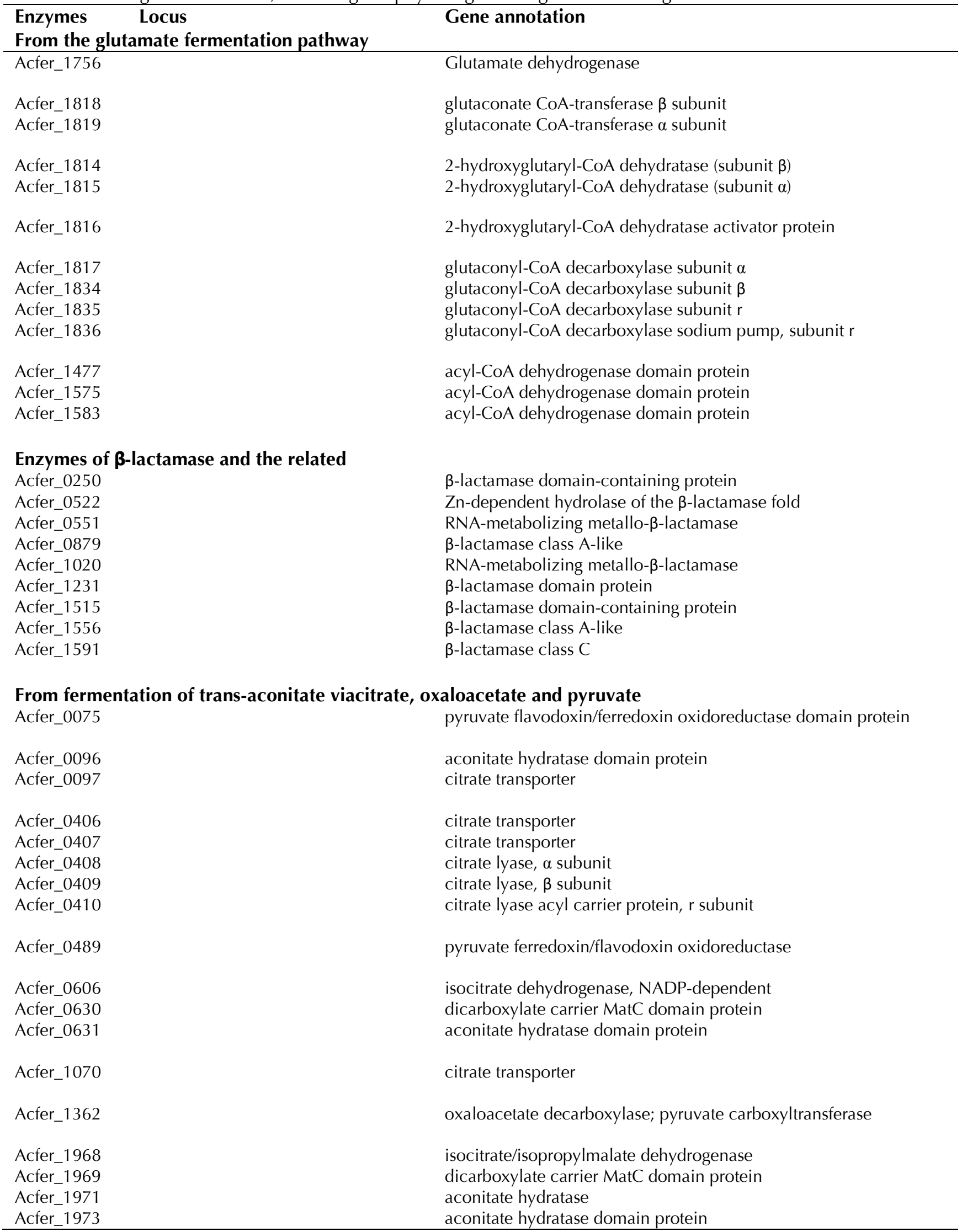


The citrate fermentation via oxaloacetate and pyruvate is another important pathway by which $A$. fermentans $\mathrm{VR} 4^{\mathrm{T}}$ is able to utilize trans-aconitate and citrate as an energy source $[4,28]$. Genes responsible for this processing tend to cluster in both genomes. Unlike the case of glutamate fermentation, genes within the trans-aconitate and citrate fermentation pathway exhibit a distinct organization in the two genomes (data not shown). This might imply differences in gene regulation or in substrate uptake. Table 6 lists major genes identified from the fermentation pathways discussed above.

\section{Acknowledgements}

We would like to gratefully acknowledge the help of Gabriele Gehrich-Schröter for growing A. fermentans cultures and Susanne Schneider for DNA extraction and quality analysis (both at DSMZ). This work was performed under the auspices of the US Department of Energy Office of Science, Biological and Environmental Research Program, and by the University of California, Lawrence Berkeley National Laboratory under contract

\section{References}

1. Rogosa M. Acidaminococcus gen. n., Acidaminococcus fermentans sp. n., anaerobic Gramnegative diplococci using amino acids as the sole energy source for growth. I Bacteriol 1969; 98:756-766. PubMed

2. Skerman VBD, McGowan V, Sneath PHA, eds. Approved lists of bacterial names. Int J Syst Bacteriol 1980; 30:225-420. doi:10.1099/00207713$\underline{30-1-225}$

3. Fuller R. Some morphological and physiological characteristics of Gram-negative anaerobic bacteria isolated from the alimentary tract of the pig. J App/ Bacteriol 1966; 29:375-379. PubMed

4. Cook GM, Rainey FA, Chen G, Stackebrandt E, Russell JB. Emendation of the description of Acidaminococcus fermentans, a trans-aconitate- and citrate-oxidizing bacterium. Int I Syst Bacteriol 1994; 44:576-578. PubMed doi:10.1099/00207713-44-3-576

5. Jumas-Bilak E, Carlier JP, Jean-Pierre H, Mory F, Teyssier C, Gay B, Campos J, Marchandin H. Acidaminococcus intestini sp. nov. isolated from human clinical samples. Int I Syst Evol Microbiol 2007; 57:2314-2319. PubMed

doi:10.1099/ijs.0.64883-0

6. Mai V, Greenwald B, Morris JG, Jr., Raufman JP, Stine OC. Effect of bowel preparation and colonoscopy on post-procedure intestinal microbiota
Acidaminococcus has been considered highly susceptible to $\beta$-lactam antibiotics until Galán et al. [50] discovered the first $\beta$-lactamase in this species. Throughout the genomes of $A$. fermentans VR4 $^{\mathrm{T}}$ and $A$. sp. D21, there are about $10 \beta$ lactamase or $\beta$-lactamase related genes (Table 6 ). This indicates that both $A$. fermentans $\mathrm{VR}^{\mathrm{T}}$ and $A$. sp. D21 can be resistant to $\beta$-lactam antibiotics. The organism might thus contribute, via HGT, to the origin or spread of resistance genes in one of the most complex microbial ecosystems known.

No. DE-AC02-05CH11231, Lawrence Livermore National Laboratory under Contract No. DE-AC5207NA27344, and Los Alamos National Laboratory under contract No. DE-AC02-06NA25396, and Oak Ridge National Laboratory under contract No. DE-AC05000R22725, as well as German Research Foundation (DFG) INST 599/1-2.

composition. Gut 2006; 55:1822-1823. PubMed doi:10.1136/gut.2006.108266

7. Monteils V, Cauquil L, Combes S, Godon JJ, Gidenne T. Potential core species and satellite species in the bacterial community within the rabbit cecum. FEMS Microbiol Ecol 2008; 66:620-629. PubMed doi:10.1111/j.1574-6941.2008.00611.x

8. Chun J, Lee JH, Jung $Y$, Kim M, Kim S, Kim BK, Lim YW. EzTaxon: a web-based tool for the identification of prokaryotes based on $16 \mathrm{~S}$ ribosomal RNA gene sequences. Int I Syst Evol Microbiol 2007; 57:2259-2261. PubMed doi:10.1099/ijs.0.64915-0

9. Kurokawa K, Itoh T, Kuwahara T, Oshima K, Toh H, Toyoda A, Takami H, Morita H, Sharma VK, Srivastava TP, et al. Comparative metagenomics revealed commonly enriched gene sets in human gut microbiomes. DNA Res 2007; 14:169-181. PubMed doi:10.1093/dnares/dsm018

10. Lee C, Grasso C, Sharlow MF. Multiple sequence alignment using partial order graphs. Bioinformatics 2002; 18:452-464. PubMed doi:10.1093/bioinformatics/18.3.452

11. Castresana J. Selection of conserved blocks from multiple alignments for their use in phylogenetic analysis. Mol Biol Evol 2000; 17:540-552. PubMed 
12. Stamatakis A, Hoover P, Rougemont J. A rapid bootstrap algorithm for the RAxML web servers. Syst Biol 2008; 57:758-771. PubMed doi:10.1080/10635150802429642

13. Pattengale ND, Alipour M, Bininda-Emonds ORP, Moret BME, Stamatakis A. How many bootstrap replicates are necessary? Lect Notes Comput Sci 2009; 5541:184-200. doi:10.1007/978-3-64202008-7_13

14. Liolios K, Chen IM, Mavromatis K, Tavernarakis N, Hugenholtz P, Markowitz VM, Kyrpides NC. The Genomes On Line Database (GOLD) in 2009: status of genomic and metagenomic projects and their associated metadata. Nucleic Acids Res 2010; 38:D346-D354. PubMed doi:10.1093/nar/gkp848

15. LaButti K, Pukall R, Steenblock K, Glavina Del Rio T, Tice H, Copeland A, Cheng JF, Lucas S, Chen F, Nolan M, et al. Complete genome sequence of Anaerococcus prevotii type strain $\left(P C 1^{\top}\right)$. Stand Genomic Sci 2009; 1:159-165. doi:10.4056/sigs.24194

16. Gronow S, Welnitz S, Lapidus A, Nolan M, Ivanova N, Glavina Del Rio T, Copeland A, Chen F, Tice $\mathrm{H}$, Pitluck S, et al. Complete genome sequence of Veillonella parvula type strain $\left(\mathrm{Te}^{\mathrm{T}}\right)$. Stand Genomic Sci 2010; 2:57-65. doi:10.4056/sigs.521107

17. Field D. Garrity, G. Gray, T. Morrison, N. Selengut, J. Sterk, P. Tatusova, T. Thomson, N. Allen, MJ. Angiuoli, SV. The minimum information about a genome sequence (MIGS) specification. Nat Biotechnol 2008; 26:541-547. PubMed doi:10.1038/nbt1360

18. Woese CR, Kandler O, Wheelis ML. Towards a natural system of organisms: proposal for the domains Archaea, Bacteria, and Eucarya. Proc Natl Acad Sci USA 1990; 87:4576-4579. PubMed doi:10.1073/pnas.87.12.4576

19. Garrity GM, Holt JG. The road map to the manual. In: Garrity GM, Boone DR, Castenholz RW (eds), Bergey's Manual of Systematic Bacteriology, Second Edition, Vol. 1, Springer, New York, 2001, p. 119-169.

20. Gibbons NE, Murray RGE. Proposals concerning the Higher Taxa of Bacteria. Int I Syst Bacteriol 1978; 28:1-6. doi:10.1099/00207713-28-1-1

21. Marchandin $\mathrm{H}$. Teyssier, Campos J, Jean-Pierre $\mathrm{H}$, Roger F, Gay B, Carlier JP, Jumas-Bilak. Negativicoccus succinicivorans gen. nov., sp. nov., isolated from human clinical samples, emended description of the family Veillonellaceae and de- scription of Negativicutes classis nov., Selenomonadales ord. nov. and Acidaminococcaceae fam. nov. in the bacterial phylum Firmicutes. Int J Syst Evol Microbiol 2010; 60:1271-1279. PubMed doi:10.1099/ijs.0.013102-0

22. Rogosa M. Genus 11. Acidaminococcus. In: NR Kreig and JG Holt (eds.), Bergey's manual of systematic bacteriology, Vol. 1. The Williams and Wilkins Co., Baltimore, 1984, 684.

23. Rogosa M. Transfer of Veillonella Prévot and Acetaminococcus Rogosa from Neisseriaceae to Veillonellaceae fam. nov. and the inclusion of Megasphaera Rogosa in Veillonellaceae. Int J Syst Bacteriol 1971; 21:231-233. doi:10.1099/00207713-21-3-231

24. Classification of Bacteria and Archaea in risk groups. http://www.baua.de TRBA 466

25. Ashburner M. Ball, CA. Blake, JA. Botstein, D. Butler, H. Cherry, JM. Davis, AP. Dolinski, K. Dwight, SS. Eppig, JT. Gene ontology: tool for the unification of biology. The Gene Ontology Consortium. Nat Genet 2000; 25:25-29. PubMed doi:10.1038/75556

26. Härtel $U$, Buckel W. Fermentation of transaconitate via citrate, oxaloacetate, and pyruvate by Acidaminococcus fermentans. Arch Microbiol 1996; 166:342-349. PubMed doi:10.1007/s002030050393

27. Härtel U, Buckel W. Sodium ion-dependent hydrogen production in Acidaminococcus fermentans. Arch Microbiol 1996; 166:350-356. PubMed doi:10.1007/s002030050394

28. Cook GM, Russell JB. Dual mechanisms of tricarboxylate transport and catabolism by Acidaminococcus fermentans. Appl Environ Microbiol 1994; 60:2538-2544. PubMed

29. Hans M, Buckel W, Bill E. The iron-sulfur clusters in 2-hydroxyglutaryl-CoA dehydratase from Acidaminococcus fermentans. Biochemical and spectroscopic investigations. Eur J Biochem 2000; 267:7082-7093. PubMed doi:10.1046/j.14321327.2000.01809.x

30. Both B, Buckel W, Kroppenstedt R, Stackebrandt E. Phylogenetic and chemotaxonomic characterization of Acidaminococcus fermentans. FEMS Microbiol Lett 1992; 97:7-11. doi:10.1111/j.15746968.1992.tb05431.x

31. White DC, Ringelberg DB. Utility of the signature lipid biomarker analysis in determining in situ viable biomass, community structure and nutritional/physiological status of deep subsurface micro- 
biota. In: The Microbiology of the terrestrial deep subsurface (Amy PS, Haldeman D, eds). 1997. LCRC Press, New York, pp 119-136.

32. Klenk HP, Göker M. En route to a genome-based classification of Archaea and Bacteria? Syst Appl Microbiol 2010; 33:175-182. PubMed doi:10.1016/j.syapm.2010.03.003

33. Wu D, Hugenholtz P, Mavromatis K, Pukall R, Dalin E, Ivanova N, Kunin V, Goodwin L, Wu M, Tindall BJ, et al. A phylogeny-driven genomic encyclopedia of Bacteria and Archaea. Nature 2009; 462:1056-1060. PubMed doi:10.1038/nature08656

34. List of growth media used at DSMZ: http://www.dsmz.de/microorganisms/media_list.p hp.

35. Sims D, Brettin T, Detter JC, Han C, Lapidus A, Copeland A, Glavina Del Rio T, Nolan M, Chen $\mathrm{F}$, Lucas $\mathrm{S}$, et al. Complete genome sequence of Kytococcus sedentarius type strain $\left(541^{\mathrm{T}}\right)$. Stand Genomic Sci 2009; 1:12-20. doi:10.4056/sigs.761

36. Hyatt D, Chen GL, Locascio PF, Land ML, Larimer FW, Hauser LJ. Prodigal: Prokaryotic Dynamic Programming Genefinding Algorithm. BMC Bioinformatics 2010; 11:119. PubMed doi:10.1186/1471-2105-11-119

37. Pati A, Ivanova N, Mikhailova N, Ovchinikova G, Hooper SD, Lykidis A, Kyrpides NC. GenePRIMP: A gene prediction improvement pipeline for microbial genomes. Nat Methods 2010; 7:455-457. PubMed doi:10.1038/nmeth.1457

38. Markowitz VM, Mavromatis K, Ivanova NN, Chen IMA, Chu K, Kyrpides NC. Expert IMG ER: A system for microbial genome annotation expert review and curation. Bioinformatics 2009; 25:22712278. PubMed doi:10.1093/bioinformatics/btp393

39. Müller U, Buckel W. Activation of (R)-2hydroxyglutaryl-CoA dehydratase from Acidaminococcus fermentans. Eur J Biochem 1995; 230:698-704. PubMed doi:10.1111/j.14321033.1995.tb20611.x

40. Hans M, Bill E, Cirpus I, Pierik AJ, Hetzel M, Alber D, Buckel W. Adenosine triphosphateinduced electron transfer in 2-hydroxyglutarylCoA dehydratase from Acidaminococcus fermentans. Biochemistry 2002; 41:5873-5882. PubMed doi:10.1021/bi020033m
41. Buckel W. Sodium ion-translocating decarboxylases. Biochim Biophys Acta 2002; 1505:15-27.

42. Locher KP, Hans M, Yeh AP, Schmid B, Buckel W, Rees DC. Crystal structure of the Acidaminococcus fermentans 2-hydroxyglutaryl-CoA dehydratase component A. J Mol Biol 2001; 307:297308. PubMed doi:10.1006/jmbi.2000.4496

43. Braune A, Bendrat K, Rospert S, Buckel W. The sodium ion translocating glutaconyl-CoA decarboxylase from Acidaminococcus fermentans: cloning and function of the genes forming a second operon. Mol Microbiol 1999; 31:473-487.

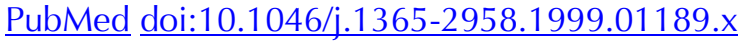

44. Buckel W, Dorn U, Semmler R. Glutaconate CoA-transferase from Acidaminococcus fermentans. Eur J Biochem 1981; 118:315-321. PubMed doi:10.1111/j.1432-1033.1981.tb06404.x

45. Mack M, Bendrat K, Zelder O, Eckel E, Linder D, Buckel W. Location of the two genes encoding glutaconate coenzyme A-transferase at the beginning of the hydroxyglutarate operon in Acidaminococcus fermentans. Eur J Biochem 1994;

226:41-51. PubMed doi:10.1111/j.14321033.1994.tb20024.x

46. URL for MetCyc pathway: http://biocyc.org/META/newimage? type $=$ PATHWAY \&object $=$ P162PWY\&detail-level=2\&ENZORG=TAX-905

47. URL for IMG-GEBA. http://img.jgi.doe.gov/cgibin/geba/main.cgi

48. Shrestha PM, Noll M, Liesack W. Phylogenetic identity, growth-response time and rRNA operon copy number of soil bacteria indicate different stages of community succession. Environ Microbiol 2007; 9:2464-2474. PubMed doi:10.1111/j.1462-2920.2007.01364.x

49. Koonin EV, Makarova KS, Aravind L. Horizontal gene transfer in prokaryotes: quantification and classification. Annu Rev Microbiol 2001; 55:709742. PubMed doi:10.1146/annurev.micro.55.1.709

50. Galán JC, Reig M, Navas A, Baquero F, Blázquez J. ACl-1 from Acidaminococcus fermentans: characterization of the first $\beta$-lactamase in anaerobic cocci. Antimicrob Agents Chemother 2000; 44:3144-3149. PubMed doi:10.1128/AAC.44.11.3144-3149.2000 